$\xi=-1$

\title{
Improving the Vehicular Networks (Vanet) Qos through Various Routing Algorithm - a Survey
}

\author{
${ }^{1}$ M.Newbegin, ${ }^{2}$ Dr.A.Rengarajan \\ ${ }^{1}$ Research Scholar, Bharathiar University. \\ ${ }^{2}$ Professor, Veltech Multitech Dr.Rangarajan Dr.Sakunthala Engineering College. \\ *Corresponding authorE-mail: newbegin_m@yahoo.com
}

\begin{abstract}
Vehicular Ad hoc Network (VANET), a subclass of portable Ad Hoc arranges (MANETs), is a promising methodology for future wise transportation framework. The principle distinction amongst VANET and MANET is the unique portability design and quickly alterable topology. Because of a ton of aggravations in city condition, the greater part of the current convention neglects to give more prominent effectiveness. This article gives a thorough investigation of MANET and VANET, its qualities, the directing conventions and the correlation of both progressive systems. The rising and a testing convention ABSTAR is talked about in detail with the correlation of couple of other directing calculation to discover the throughput esteems and keep away from the bundle drop. Planned particularly for execution change in exceptionally portable condition of VANETs (city condition).
\end{abstract}

Keywords: Vanet, Manet, Gps.

\section{Introduction}

A Vehicular Ad-hoc Network (VANET) is a sort of Mobile Adhoc Network (MANET) that is utilized to give interchanges between adjacent vehicles and amongst vehicles and settled framework on the roadside. Vehicular Ad hoc Network (VANET), a subclass of versatile Ad Hoc organizes (MANETs), is a promising methodology for future insightful transportation framework (ITS). VANET is a kind of MANET yet the directing conventions of MANET are not plausible with VANET. Vehicular Ad hoc Network (VANET) as an extraordinary class of Mobile Ad hoc Network (MANET) contrasts from the last in various parameters [3]. The primary distinction between V ANET and MANET is the exceptional versatility design and quickly alterable topology. Giving more noteworthy proficiency in city condition has an extraordinary part in Intelligent Transportation System (ITS). A portable specially appointed system is a gathering of at least two hubs furnished with remote interchanges and systems administration capacities without focal system control, which might be alluded to as a foundation less portable network[4].VANETs is developing as another innovation.

VANETs are a remote specially appointed system which furnishes correspondences with DSRC (Dedicated short range interchanges) among vehicles and street side (RSU) settled equipment's. There are two sorts of interchanges in VANETs. Right off the bat vehicle to vehicle (V2V) correspondence in which vehicles (hubs) that is only the information can be exchange between each other with no settled foundation. Vehicle to framework (V2I) in which vehicles (hubs) sends or gets information to/from street side unit[4]. VANETs assumes a noteworthy part in Intelligent Transportation System (ITS) so as to give security and solace to the street clients with the assistance of VANETs. It is a critical class of smart transportation framework in which the hubs are outfitted with Dedicated Short Range Communication (DSRC) to trade information [4]. The hubs in a VANET use On Board Units (OBUs) to speak with each other either straightforwardly or with the assistance of a middle of the road hub, for example, switch or Road Side Unit (RSU) [3].

Vehicular Ad-hoc Networks (VANETs) speak to a quickly developing, especially difficult class of Mobile Ad Hoc Networks (MANETs) . Vehicular impromptu systems (VANETs) are made by applying the standards of versatile specially appointed systems (MANETs) - the unconstrained formation of a remote system for information trade - to the area of vehicles. VANETs were first said and presented "auto to-auto specially appointed versatile correspondence and systems administration" applications, where systems can be framed and data can be handed-off among autos. It was demonstrated that vehicle-to-vehicle and vehicle-to-roadside interchanges models will exist together in VANETs to give street security, route, and other roadside administrations. VANETs are a key piece of the canny transportation frameworks (ITS) system. Once in a while, VANETs are alluded as Intelligent Transportation Networks . Auto-versatile businesses are to a great extent joining forces through a few continuous research activities to set up models for VANETs.

\section{Localisation Technique in Vanet}

Confinement is a noteworthy issue in VANET the same number of its applications incorporate position information. The regular localisation methods in it utilize Global Positioning System (GPS) recipients and movement sensors. GPS is broadly utilized for directing and route restriction notwithstanding having some precision issues. Be that as it may, GPS recipients unquestionably are not the best alternative for circumstances where there is poor perceivability of satellites like indoor or thick urban zones [3]. 
a) Global Positioning System: GPS technology became a reality through the efforts of the American military, which established a satellite-based navigation system consisting of a network of 24 satellites orbiting the earth. GPS is also known as the NAVSTAR (Navigation System for Timing and Ranging).GPS works all across the world and in all weather conditions, thus helping users track locations, objects, and even individuals. GPS or Global Positioning System is a satellite route framework that outfits area and time data in all atmosphere conditions to the client. GPS is utilized for route in planes, boats, autos and trucks too. GPS innovation can be utilized by any individual in the event that they have a GPS collector. GPS has turned into a proficient instrument in the field of logical utilize, business, reconnaissance and following. GPS is utilized with the exception of in areas where it is hard to identify the flag for instance, submerged, underground area, inside the building and gives in. The GPS does not require the client to transmit any information, and it works autonomously of any telephonic or web gathering, however these innovations can upgrade the convenience of the GPS situating data. The GPS gives basic situating capacities to military, common, and business clients around the globe. GPS can be utilized as a part of two noteworthy applications a) Civilian Applications b) Military Applications. GPS consist of 3 fragments comprise of Space, client and control portions.

b) Space segment - The satellites are the core of the Global situating framework which finds the situation by communicating the flag utilized by the beneficiary. The signs are blocked when they go through structures, mountains, and individuals. To compute the position, the signs of four satellites ought to be bolted.

c) User segment - This portion incorporates military and regular citizen clients. It includes a delicate collector which can distinguish signals (energy of the flag to be not as much as a quadrillionth energy of a light) and a PC to change over the information into valuable data. GPS recipient finds your own position however forbids you being followed by another person.

d) Control segment - This encourages the whole framework to work productively. It is fundamental that the transmission signals must be refreshed and the satellites ought to be kept in their fitting circles.

\section{Wireless Localisation}

Remote confinement is one of the methods that can be utilized to distinguish counterfeit area promotions. They utilize the stationary base stations, otherwise known as Road Side Units (RSUs) situated along the edge of the streets all around the guide in VANETs, to play out the restriction. These RSUs have a wide radio range that enables the protection instrument to have the capacity to screen a considerable measure of vehicles in the meantime. They can tune in to VANET messages and they are altogether associated with each other by wired associations through the framework. Gotten Signal Strength Indicator (RSSI) ascertained from the transmissions of these messages.

a) Dead Reckoning: Dead retribution, a system used to locate their present position utilizing the past decided position. the words dead retribution are likewise used to mean the way toward evaluating the estimation of any factor amount by utilizing a prior esteem and including whatever progressions have happened meanwhile.

Frequently, this utilization suggests that the progressions are not known accurately [3]. The prior esteem and the progressions might be estimated or computed amounts. The exactness of dead retribution can be expanded fundamentally by utilizing other, more dependable strategies to get another fix part path through the excursion.

b) Cellular Localization: In urban situations, it takes help of portable cell foundation to assess the situation of the protest. Portable cell framework requires correspondence foundation made out of cell base stations. Cell phones in a city continue changing their base station when flag quality from new base station is more prominent (known as handoff method) [3].

c) Map Matching: A few positions were gotten over normal interims of time to make assessed direction. At that point assessed direction is contrasted with the known computerized guide to locate the most reasonable way geometry on the guide that matches direction. This strategy can be utilized to identify the exact position data.

d) Image/video Processing: Picture/video handling likewise be utilized for restriction purposes. Many places cameras are as of now accessible in security frameworks e.g., parking garages and held spots. These philosophies were prepared and nourish to information combination calculations to assess and foresee a vehicle's area.

\section{Comparision of Manet and Vanet}

Like versatile impromptu systems (MANETs), hubs in VANETs self-sort out and self-oversee data in an appropriated form without a concentrated specialist or a server directing the correspondence [2].MANET hubs require impromptu compose steering conventions. They are of two kind's viz. table driven steering conventions and on request directing conventions. In VANET, the correspondence hubs are preceding onward pre-characterized streets as concluded initially. These systems are utilized for correspondence between following:

MANET: In specially appointed systems every one of the hubs are versatile in nature and subsequently they can be interfaced powerfully in subjective mold.

\begin{tabular}{|c|c|c|}
\hline \multirow{7}{*}{$\begin{array}{c}\text { MANET } \\
\text { VS } \\
\text { VANET }\end{array}$} & MANET & VANET \\
\hline & Between Vehicles & $\begin{array}{l}\text { Between Vehicles and } \\
\text { road side }\end{array}$ \\
\hline & $\begin{array}{l}\text { Worksin dynamic } \\
\text { Topologies }\end{array}$ & Hybrid topology \\
\hline & $\begin{array}{l}\text { Variable capacity } \\
\text { Links }\end{array}$ & Nodes are predefined \\
\hline & $\begin{array}{l}\text { Energy Constrained } \\
\text { Operation }\end{array}$ & Pure Ad-Hoc network \\
\hline & $\begin{array}{l}\text { Limited Physical } \\
\text { Security }\end{array}$ & $\begin{array}{l}\text { Unbounded network } \\
\text { size }\end{array}$ \\
\hline & $\begin{array}{l}\text { Limited in information } \\
\text { exchange }\end{array}$ & $\begin{array}{l}\text { Frequent exchange of } \\
\text { Information }\end{array}$ \\
\hline
\end{tabular}

Fig 1: General Comparision between Vanet and Manet

\subsection{Secure Data Transmission}

Information can forward through accessible ways. Information is part into $\mathrm{n}$ number of pieces and sent in different ways safely by scrambling and mark checking in each bounce. This memory devouring procedure is outsourced to the potential vehicular cloud made out of separated vehicle in stopping spaces. Those vehicles utilize mystery keys shared for encryption, signature era and forward the information to the middle of the road hub which will then encode the information with mark utilizing by a special key and forward to the following transitional in the way. The following hub can decipher the information and outsource the sign check and re-encryption procedure to the vehicular cloud. A similar procedure will be rehashed as above in the middle of the road hub lastly dispatched to the goal. Information is sending to 
next hub in way that hub will haphazardly choose a stopping vehicle and sends the information to the vehicle.

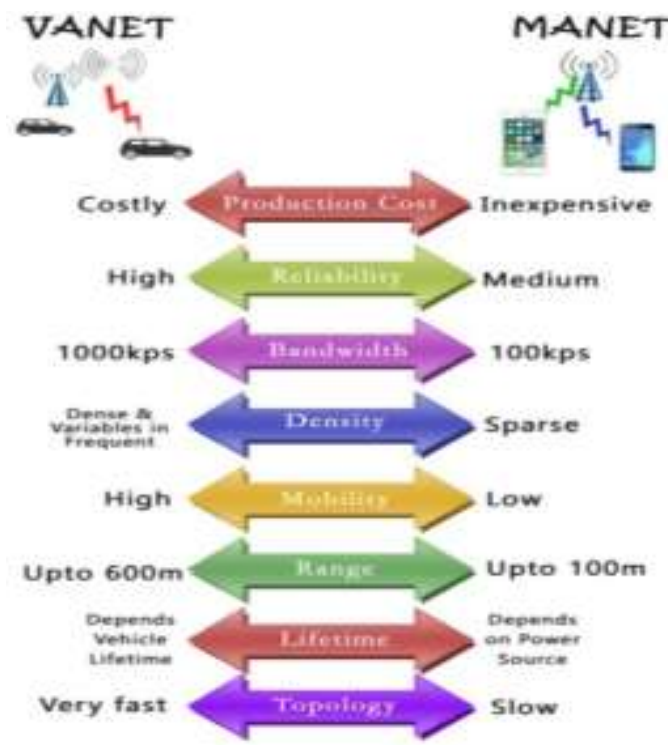

Fig 2: Comparision of Vanet with Manet

The routing protocols of MANET are not feasible to be used in the VANET network

The VANET architecture consists of three type of categories as mentioned below:

1. Cellular and WLAN network

2. Pure Ad hoc (network between vehicles and fixed gateways)

3. Hybrid(combination of both infrastructure and adhoc networks)

Moreover the communication nodes are moving on pre-defined roads as finalized

\section{Characteristics of Vanet}

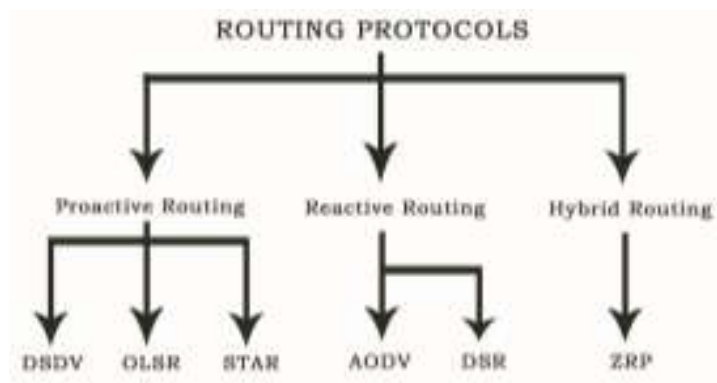

Fig 3: Routing protocols in MANET

\subsection{Optimized Link State Routing Protocols (OLSR)}

Advanced Link State Routing Protocols (OLSR) is proactive and point-to-point steering convention in light of the customary connection state algorithm [9]. It is utilizing a system called multipoint transferring for advanced message and flooding process for course setup or course support. The calculation limit the quantity of dynamic transfers for covering the neighbors and it is called Multi-Point Relays (MPR). The convention presented for exactness and security for directing the information in arrange [9].Optimized Link State Routing convention (OLSR) has two key ideas, Multipoint Relays (MPRS) calculation and Optimized State is among one - jump neighbors and cover two-bounce neighbors or sending join state data for support of steering. Each hub gets refreshes just once and unselected bundles can't retransmit refreshes. The significant preferred standpoint of this convention is the all courses and goals are known and kept up before the activity. Then again, the hubs are moving quick, because of computation of ideal hub might be incomprehensible in some cases [9].

\section{Proposed Technique}

Effective Routing is one of the key principle and success for VANETs (Vehicular Ad Shorter distances to the destination) to the hoc networks. City environment routing is one of the major challenging tasks in routing topology. Many routing topologies have been discussed in the recent days to bring the network in a smarter way. Among them Geographic Source Routing (GSR) and Anchor Bus based Street and Traffic Aware Routing protocol. ASTAR were plays a major role. All routing protocols have a lot of limitations and condition in the process. These two protocols work much better in a toughest situation to overcome the limitations. ABSTAR is a new geographical routing protocol for city environment. It works like the GPS and aims effective relay data in the network. It considers the real time road traffic variation and city environment characteristics. It monitors the vehicle speeds and the direction in the multilane and bidirectional roads. The major objective of ABSTAR is to utilize the network resources by limiting the control messages over head and transmits the data packets from source to destination in VANET with a reduced end to end delay.

1) ABSTAR Protocol: ABSTAR (Anchor Bus based Street and Traffic Aware Routing) is another crossing point based topographical steering convention competent to discover vigorous courses inside city conditions. At the end of the day we can state that the recently proposed convention ABSTAR is an improved and adjusted form of ASTAR [8]. ABSTAR additionally contains grapple based steering approach with road mindfulness and the remarkable idea of ABSTAR is to pick a course with less quantities of transports as could be expected under the circumstances. ABSTAR acquires every one of the properties of ASTAR [8] alongside some new extra highlights. In ABSTAR, the parcel is gone through different intersections so as to achieve its goal [4]

This intersection choice is a dynamic occasion and is chosen one by one (all together) in light of continuous vehicular movement variety. The sending vehicle or a middle vehicle in an intersection will choose its next goal intersection by finding the situation of the neighbouring intersections with the assistance of map[4]. A Score is figured to every intersection by considering the movement thickness $\mathrm{Hj}$ (it is a measure of the activity thickness between the present crossing point and potential convergence $j$. boulevards with higher thickness is favored) and the bend metric Distance Lj. The best goal intersection $\mathrm{j}$ is then the intersection with the most astounding score

$\mathrm{V}(\mathrm{j})=\beta \times \mathrm{f}\left(\mathrm{H}_{\mathrm{j}}\right)+\alpha \mathrm{Xg}(\mathrm{Lj})$,

Where $\beta$ and $\alpha$ are weighting factors. $f$ and $g$ are unspecified characters. After determination of goal intersection, the information parcels must be sent towards the chose goal intersection by utilizing enhanced ravenous system. Once the goal intersection is resolved, the enhanced insatiable procedure is utilized to forward bundles towards the chose intersections. ABSTAR utilizes statically and powerfully appraised maps to locate the quantity of Junctions[4]. ABSTAR gather the most recent data of movement with a specific end goal to discover the way. The whole vehicle keeps up a different neighbour table in which speed, position and bearing

2) Recovering the packets: ABSTAR utilizes another recuperation technique. At the point when a parcel meets issue to go from an intersection, that intersection is set apart as "out of administration". So different parcels won't utilize that intersection until the point that that intersection's status 
changes to "operational state". In the event that any intersection is out of request, the data about that specific intersection is passed to every vehicle in the system and every vehicle will refreshes their directing data and city maps by denoting that put in as out of request. In this manner no vehicle will utilize that intersection to achieve its goal.

3) Performance Evaluation: The performance ABSTAR in Vanet can be checked in two ways. Checking the throughput and the packet drop metrics with some other routing protocols. It is very much used to check the effectiveness of the VANET routing protocols, the performance of protocol deliver and the algorithm for a routing protocol performs in order to discover the route towards destination.

4) Throughput: Throughput is the normal number of effectively conveyed information parcels on a correspondence system or system hub. Throughput is portrayed as the aggregate number of got parcels at the goal

5) Packet Drop: Packet drop shows total number of data packets that could not reach destination successfully. The reason for packet drop may arise due to faulty hardware, congestion and queue overflow etc. packet drop affects the network performance by consuming time and more bandwidth to resend a packet. Lower packet drop rate shows higher protocol performance[4].

\begin{tabular}{|c|c|c|c|c|}
\hline PROTOCOLS & SAR & $\overline{\text { OLSR }}$ & ABSTAR & STAR \\
\hline Position based /Geographic & & $\sqrt{ }$ & $\sqrt{ }$ & $\sqrt{ }$ \\
\hline Street aware & $\sqrt{ }$ & $\sqrt{ }$ & $\sqrt{ }$ & $\sqrt{ }$ \\
\hline Position - Junction routes & $\sqrt{ }$ & $\sqrt{ }$ & $\sqrt{ }$ & $\sqrt{ }$ \\
\hline Route- repair or recovery & $\sqrt{ }$ & $\sqrt{ }$ & $\sqrt{ }$ & $\sqrt{ }$ \\
\hline Transport route required & $x$ & $x$ & $\sqrt{ }$ & $x$ \\
\hline $\begin{array}{l}\text { Route } w \text { ith smaller number } \\
\text { of Bus }\end{array}$ & $\mathbf{x}$ & $\mathbf{x}$ & $\sqrt{ }$ & $\mathbf{x}$ \\
\hline Carry and Forward & $\sqrt{ }$ & $x$ & $\sqrt{ }$ & $x$ \\
\hline Traffic aware & $\mathbf{x}$ & $\mathbf{x}$ & $\sqrt{ }$ & $\sqrt{ }$ \\
\hline $\begin{array}{c}\text { Number of buses } \\
\text { through route is required }\end{array}$ & $x$ & $x$ & $\sqrt{ }$ & $x$ \\
\hline
\end{tabular}

Fig 4: Comparision of ABSTAR with other routing techniques

In the fig 4 - table the features can be compared with some of the routing techniques. ABSTAR is very efficient in all categories when compared to all other mentioned protocols. ABSTAR is one among the best routing protocol in the mechanism of VANET. It improves the through put quality and avoids the packet loss in the transmission flow. Figure 5 and Figure 6 clearly explains about the throughput quality and the decrement in packet loss comparison.

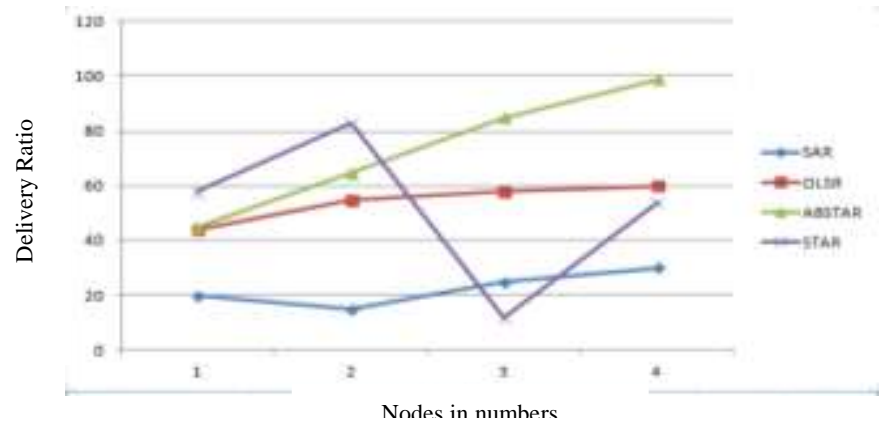

Fig 5: Increment in Throughput

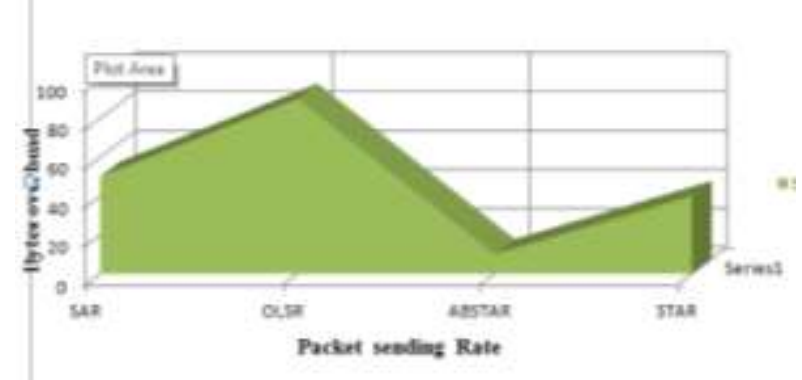

Fig 6: Packet drop (Decrement)

\section{Conclusion}

This paper present a state of the art survey in in vehicular ad hoc network (VANET) \& Mobile ad hoc network (MANET) which is a promising technology for intelligent transportation system (ITS). The above comparative study clearly explains the QoS among the various routing protocols especially ABSTAR with few routing protocols. The detailed study of MANET and VANET helps to understand the network topologies in a efficient way. ABSTAR improved performance in throughput, packet drop and routing overhead metrics. ABSTAR gives a Quality of Service compared to the existing techniques.

\section{References}

[1] VANETs Challenges and Perspectives Saleh Yousefi', MAhmoud Siadat MIousavi2 Mahmood Fathy' 2006 6th International Conference on ITS Telecommunication Proceedings.

[2] Comparative Study of VANET and MANET Routing Protocols Prabhakar Ranjan1, Kamal Kant Ahirwar2

Department of Computer Science \& Engineering ,Amity University ,Noida.

[3] Clustering in MANET and VANET: A Survey, Manu Sood, Shivani Kanwar, Department of Computer Science , 2014 International Conference on Circuits, Systems, Communication and Information Technology Applications (CSCITA).

[4] ABSTAR: Improves Qos for City Environment in VANET Anchala Arneena Moharnrnadi, Student Member, IEEE, Raj G.S, Member, IEEE and Virnal Karthick.R Raj G.S, Member, IEEE.

[5] B. Ducourthial, Y. Khaled, and M. Shawky, "Conditional transmissions: Performance study of a new communication strategy in VANET," IEEE Trans. Veh. Technol., vol. 56, no. 6, pp. 33483357, 2007.

[6] S. R. Kolte ad M. S. Madankar, "Adaptive congestion control for transmission of safety messages in VANET," 2014 Int. Conf. Converg. Technol. I2CT 2014, pp. 1-5, 2014.

[7] Topology based routing protocols for Vanet and their comparision with Manet,Kashif naseer qureshi, Abdul hanan Abdull 